\title{
RESONANT STATES IN A ONE-DIMENSIONAL QUANTUM SYSTEM
}

${ }^{1}$ Tanimu, A. and ${ }^{1,2}$ Bagudo, I. M.

${ }^{1}$ Department of Physics Umaru Musa Yar'adua University Katsina

${ }^{2}$ Ibrahim Shehu Shema Centre for Renewable Rnergy and Research Umaru Musa Yar'adua University Katsina Corresponding Author's email: abdullahi.tanimu@umyu.edu.ng

\begin{abstract}
In this work, the concept of resonant states (RSs) in a finite square quantum well is presented. We first derive the analytic secular transcendental equations for even and odd states by applying the outgoing wave boundary conditions into the one-dimensional Schrödinger's wave equation. The complex solution of these equations is found using the numerical Newton-Raphson method implemented in MATLAB. We can see in particular, that the RSs present a general class of Eigenstates, which includes bound states, anti-bound states, and normal RSs.
\end{abstract}

Keywords: Boundary conditions, Eigenstates, Complex solution, Schrödinger's equation, MATLAB.

\section{INTRODUCTION}

Resonant States (RSs) have been known in quantum mechanics for a quite long time, since the pioneering work of Gamow, 1928 and Siegert, 1939. Theyappear, in the form of resonances, in almost every field of Physics, from classical mechanics and electrodynamics to quantum physics and gravity. Despite this fact, however, many fundamental aspect are still to be investigated. Also, resonant phenomena are of increasing importance in quantum mechanics especially given rapid progress in the physics of semiconductor nanostructures which can be described by various types of quantumpotentials. Many textbooks (Mandle, 2010) describe quantum resonances as singularities of theS-matrix. This is equivalent to solving the Schrödinger equationwith outgoing wave boundary conditions (Doost et al, 2012, Muljarov et al, 2010, Siegert, 1939). These boundary conditions strictly define RSs. These states have complex energy eigenvaluescausing them to decay exponentially in time, leaking out of the system (quantum well/barrier). There are numerous ideas as to how to investigate RSs in quantum- mechanical systems, however, there are certain problems to be overcome, such as knowing how the potential in the Schrödinger equation gives rise to resonances and how to treat and interpret them (Hatano, 2008). New method of finding RSs in an arbitrary called resonant-state expansion (RSE) has recently been introduced (Armitage et al, 2014, Doost et al, 2012, Muljarov et al, 2010). In this work, the concept of RSs in a square quantum well is presented. We first obtain the analytic secular equations in terms of even and odd states by applying the outgoing wave boundary conditions. These equations are solved numerically using the NewtonRaphson procedureimplemented in MATLAB. We consider all types of states (bound, anti-bound, and normal RSs) in such a system. We also calculated the wave functions of RSs.

\section{THEORY}

\section{The formalism of Resonant States (RSs)}

The quantum-mechanical system we use in this work is described by a one-dimensional Schrödinger equation with a finite square well potential. We use this potential because of its simplicity and of practical importance for low dimensiona structures such as quantum wells (Andrew, 2010). Quite generally, non-relativistic Schrödinger equation (Tanimu and Muljarov, 2018) for an arbitrary particle in a three-dimensional potential is

$$
H(r) \psi_{n}(r)=E_{n} \psi_{n}(r)
$$

The wave functions of RSs satisfy the outgoing waves boundary condition at $r \rightarrow \infty$. For a constant potential, this means that $\psi_{n}(x \rightarrow$ $\pm \infty) \propto e^{i k|x|}$.A one-dimensional form of Eq.(1)i.e. the timeindependent Schrödingerequation takes the form:

$$
\left[-\frac{\hbar^{2}}{2 m} \frac{d^{2}}{d x^{2}}+V(x)\right] \psi_{n}(x)=E_{n} \psi_{n}(x)
$$

Where $E_{n}=\frac{\hbar^{2} k_{n}^{2}}{2 m}$ is the energy of theparticle. For convenience, we use in the following $m=1 / 2$ and $\hbar=1$, so that $E_{n}=k_{n}^{2}$, where $k_{n}$ is the eigen wave number of the particle, $\hbar$ is the Planck's constant, $m$ is the effective mass of the particle.

The potential $V(x)$ of the particle is

$$
V(x)=\left\{\begin{array}{rr}
-V_{0}, & |x|<a \\
0, & |x|>a
\end{array}\right.
$$

This potential has been covered in depth by many textbooks such as (Mandle, 2010). However, RSs in general are usually not considered in textbooks. Therefore, in this work, it is interesting to see how the RSs move in the complex $k$-plane as we increase the depth of the well. The solutions to equation (2) in terms of plane waves are

$\psi_{n}(x)=\left\{\begin{array}{l}A e^{i k_{n} x}, \quad x>a \\ C e^{i \xi_{n} x}+D e^{-i \xi_{n} x}, \quad-a<x<a \\ B e^{-i k_{n} x}, \quad x<-a\end{array}\right.$

where $A, B, C$ and $D$ depends on $n$, but we drop the index for the brevity of notations and also, $k_{n}$ and $\xi_{n}$ are the wavenumbers in their respective regions and are related as 


$$
\xi_{n}=\sqrt{k_{n}^{2}+V_{0}}
$$

To find the eigenvalues, $k_{n}$ we require that from equation (4) $\psi_{n}(x)$ and $\psi_{n}^{\prime}(x)$ are continuous at $x= \pm a$. Solving $C$ and $D$ in terms of $A$ and $B$ weobtain (dropping index $n$ also, for the brevity of notations)

$$
\begin{gathered}
C=\frac{(\xi+k)}{2 \xi} e^{i(k-\xi) a} A \\
D=\frac{(\xi-k)}{2 \xi} e^{i(k+\xi) a} A
\end{gathered}
$$

and

$$
\begin{aligned}
& C=\frac{(\xi-k)}{2 \xi} e^{i(k+\xi) a} B \\
& D=\frac{(\xi+k)}{2 \xi} e^{i(k-\xi) a} B .
\end{aligned}
$$

This yield two equations for $B$ in terms of $A$

$B=\frac{(\xi+k)}{(\xi-k)} e^{-2 i \xi a} A$

and

$$
B=\frac{(\xi-k)}{(\xi+k)} e^{2 i \xi a} A
$$

which can only be satisfied if

$$
\left(\frac{\xi-k}{\xi+k}\right)^{2}=e^{4 i \xi a}
$$

There are two possible solutions to equation (12):

Solution 1 is

$$
\frac{\xi-k}{\xi+k}=-e^{2 i \xi a}
$$

And solution 2 is

$$
\frac{\xi-k}{\xi+k}=e^{2 i \xi a}
$$

which after some algebra leads to

$$
k_{n}=-i \xi_{n} \cot \left(\xi_{n} a\right)
$$

where we have restored index $n$. This solution is odd.

And $k_{n}=i \xi_{n} \tan \left(\xi_{n} a\right)$

This solution is even.

Equations (15) and (16) (secular transcendental equations) are solved together with equation (5) to find the Eigen wave numbers which are plottedin the complex $k_{n}$-plane fig. 1 .These equations cannot be solved analytically. However, in this work,we employ the use of Newton-Raphson procedure in MATLAB, which istypically fast to converge to find their complex roots which give rise toall types of states. After we found our transcendental equations we thensubstitute

$$
\frac{\xi-k}{\xi+k}= \pm e^{2 i \xi a}
$$

back into the equation giving the relations between the $A, B$, $C$, and $D$ toobtain the wavefunctions. We found that: if

$$
\frac{\xi-k}{\xi+k}=+e^{2 i \xi a}
$$

then, $C=D, A=B, \psi(-x)=\psi(x)$. The solutions are even and thewavefunctions are symmetric. If however,

$$
\frac{\xi-k}{\xi+k}=-e^{2 i \xi a}
$$

then $C=-D, A=-B, \psi(-x)=-\psi(x)$. The solutions are odd and thewavefunctions are anti-symmetric.For both the even and odd solutions the wave functions have to be normalizedbut care has to be taken during the calculations. For bound states, this is aneasy task but for the resonant states which have exponentially increasing tails, an additional term must be considered to normalize them correctly. Anouter limit is required for their normalization and is given by $R$. It is foundthat the value of $R$ can be taken arbitrarily and thus for convenience we arefree to choose the boundaries of the well as the limits of this normalization. The orthonormality condition is given as (Muljarov et al, 2010):

$$
\begin{aligned}
& \delta_{n m}{ }_{a} \\
& =\int_{-a} d x \psi_{n}(x) \psi_{m}(x) \\
& -\frac{\psi_{n}(a) \psi_{m}(a)+\psi_{n}(-a) \psi_{m}(-a)}{i\left(k_{n}+k_{m}\right)} .
\end{aligned}
$$

It can also show that this equation is suitable for the usual normalizationof bound states as it reduces to the standard normalization condition as $R$ tends to infinity the condition tends towards

$$
\delta_{n m}=\int_{-\infty}^{\infty} d x \psi_{n}(x) \psi_{m}(x)
$$


which is the standard approach to normalizing thebound states.

The numerical procedure of finding the Eigen wave numbers.

There are many numerical procedures for solving equations (15) and (16). While the equations cannot be solved analytically, they can be solved numerically up to any desired accuracy. Below are the few steps we used for the solutions: - We use the relation between $\xi_{n}$ and $k_{n}$ in eq.(5). This makes

the final equations to solve written in terms of $k_{n}$ only. - The second step defines the function $f\left(k_{n}\right)$ such that the equation we solve becomes $f\left(k_{n}\right)=0$. - The third step sets the physical parameter values. - At the fourth step, we look at the function behavior, to choose the optimal guess values for the Newton-Raphson procedure. - Lastly, the solutions for $k_{n}$ are plotted in complex $k$-plane (see fig.1), and the wave functions and energies are also calculated.

\section{RESULTS}

Eigen wave numbers of an RSs

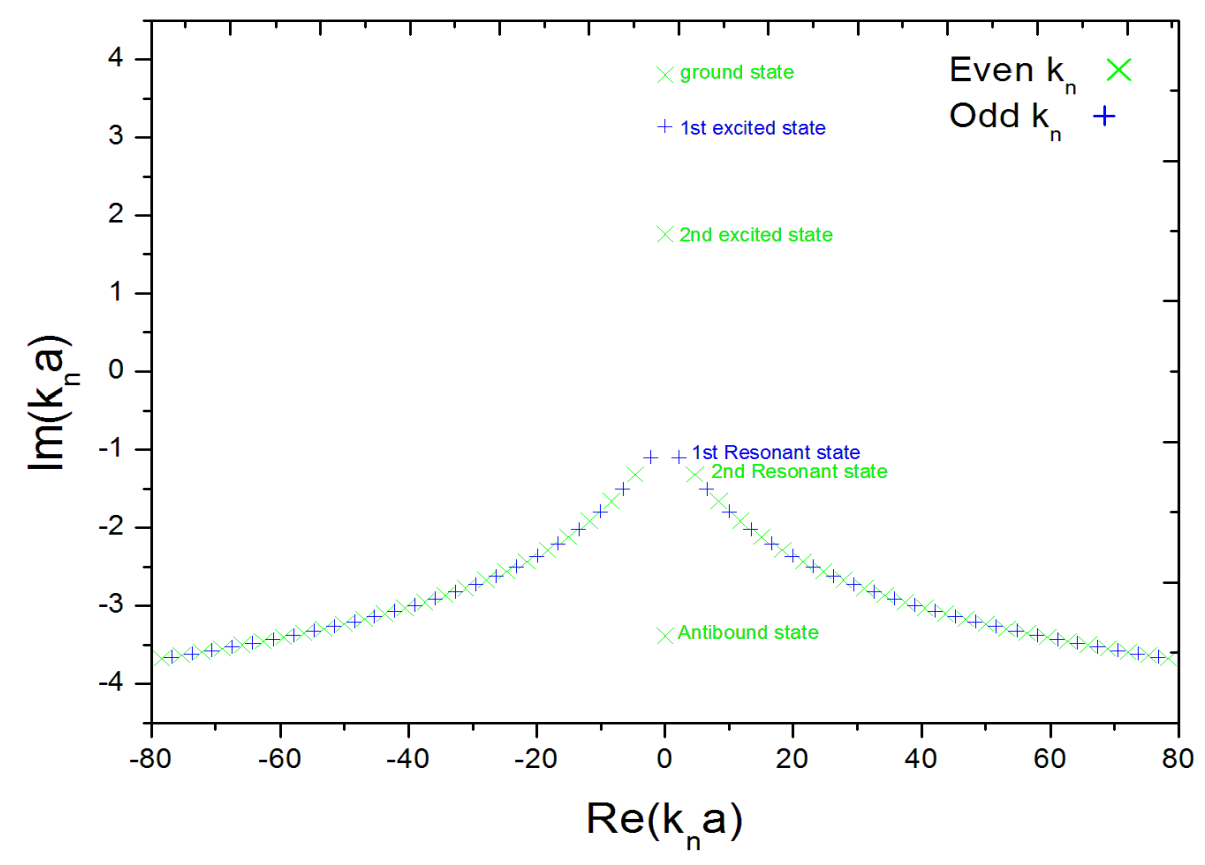

Figure 1: Solutions $k_{n}$ for the square-well potential with outgoing waves boundary conditions plotted in the complex $k_{n}$-plane. normal resonant state and corresponding anti-resonant state are

Figure 1 shows the plot of the complex eigenvalues in equations (15) and (16) which gives rise to all types of states. As we can see the resonant states present a general class of Eigen states, which includes bound states, anti-bound states, and normal resonant states. For a shallow well, ( see for example, Hatano, 2008) there is only one bound state and the $\mathrm{RSs}$ are far down in the $k$-plane almost parallel to the real axis. When we increase the depth of the well, the RSs wave numbers move upwards parallel to the imaginary axis which also leads to an increase in the number of bound states. Similar results are found in (Tanimu and Muljarov, 2018) using different parameter. When the pair of conjugate RSs hits the imaginary axis it splits up into a bound-anti-bound states pair which gets more bound when increasing the depth further. The normal

resonant states all have non-zero real and imaginary parts of $k_{n}$. Each normal resonant state with $\operatorname{Re}\left(k_{n}\right)>0$ has a partner in the negative real axis with $\operatorname{Re}\left(k_{n}\right)<0$. The positions of a symmetric concerningthe imaginary axis. Their locations are mirror images for the imaginary axis. Depending on the system parameters, there are also discrete states on the imaginary axis. These states are called bound and anti-bound states. The bound states of the system considered are the ground state, $1^{\text {st }}$ excited state, and $2^{\text {nd }}$ excited state. The bound states are located on the positive imaginary axis $\operatorname{Im}\left(k_{n}\right)>0$ while anti-bound states are located in the negative imaginary axis $\operatorname{Im}\left(k_{n}\right)<0$.

\section{Wave functions of RSs \\ Bound states}

Stationary states of a system that correspond to discrete energy levels are called bound states. For bound states the energy is real. We can see for example in (Tanimu and E. A. Muljarov, 2018) that the potential generate bound states since the solution of equation (22) have an exponentially decaying tail at the 
boundaries. For potential vanishing at $|x| \rightarrow \infty$ the bound state energies are negative (Uma, 2010). Applying the asymptotic boundary conditions, the bound states wavefunctions has the asymptotic behavior as

$$
\psi_{n}(x)= \pm e^{-i k_{n}|x|},|x| \rightarrow \infty
$$

But with $\kappa_{n}>0$

$$
k_{n}=i \kappa_{n}
$$

Therefore the bound state energy $\mathrm{E}$ is

$$
E_{n}=k_{n}^{2}=-\kappa_{n}^{2}
$$

which shows that the bound states can be presented as negative energy states.

\section{Normal RSs}

The resonant state can be defined as an Eigen state of the stationary Schrödinger equation with boundary conditions of outgoing waves

only. which is called Siegert condition (Siegert, 1939). For evaluation of resonant states, we seek a solution of equation (2) with complex energy

$$
k_{n}^{2}=\left(k_{n}^{\prime}-i k_{n}^{\prime \prime}\right)^{2}=\left(k_{n}^{\prime 2}-k_{n}^{\prime \prime 2}\right)-2 i k_{n}^{\prime} k_{n}^{\prime \prime}=E_{n}
$$

with $k_{n}^{\prime}>0$ and $k_{n}^{\prime \prime}>0$ such that the wave functions behaves asymptotically as:

$$
\psi_{n}(x)=\left\{\begin{array}{c}
e^{i\left(k_{n}^{\prime}-i k_{n}^{\prime \prime}\right) x}, x \rightarrow+\infty \\
\pm e^{-i\left(k_{n}^{\prime}-i k_{n}^{\prime \prime}\right) x}, \quad x \rightarrow-\infty
\end{array}\right.
$$

This nature of the wavefunction shows a positive energy state. We can see from figures 2 and 3 that $\left|\psi_{n}\right|$ diverges exponentially as $e^{k_{n}^{\prime \prime}|x|}$ (Tanimu and Muljarov, 2018). However, this exponential growth is conjugated by time decaying.

\section{Anti-bound states}

An anti-bound state shares similar features with the bound states and resonant states but it is called a separate type of state. Unlike bound states, they have a solution for the continuous range of energies which satisfies $E<0$. It diverges exponentially for large $|x|$ (see fig.2). The solution inside the well is similar to that of the bound state. We can see the wave function is symmetric around the origin, which indicates that there must be solutions of defined parity also for anti-bound states.

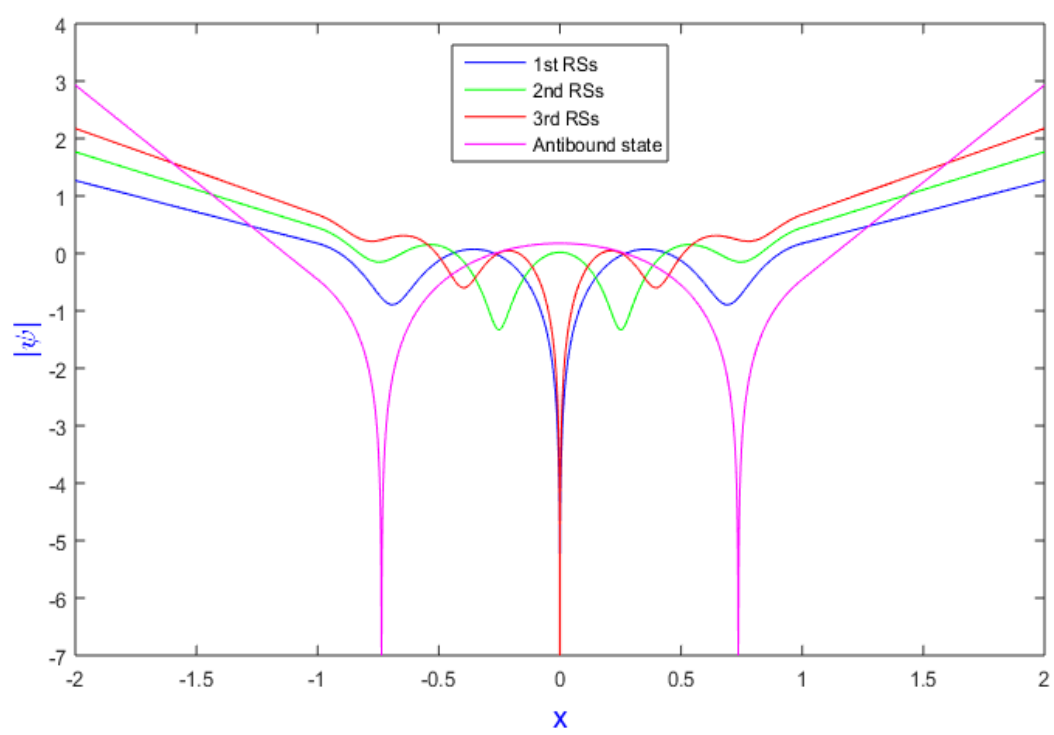

Figure 2: Plots of the absolute values of the anti-bound state, $1^{\text {st }}, 2^{\text {nd, }}$ and $3^{\text {rd }}$ resonant states wave functions. 


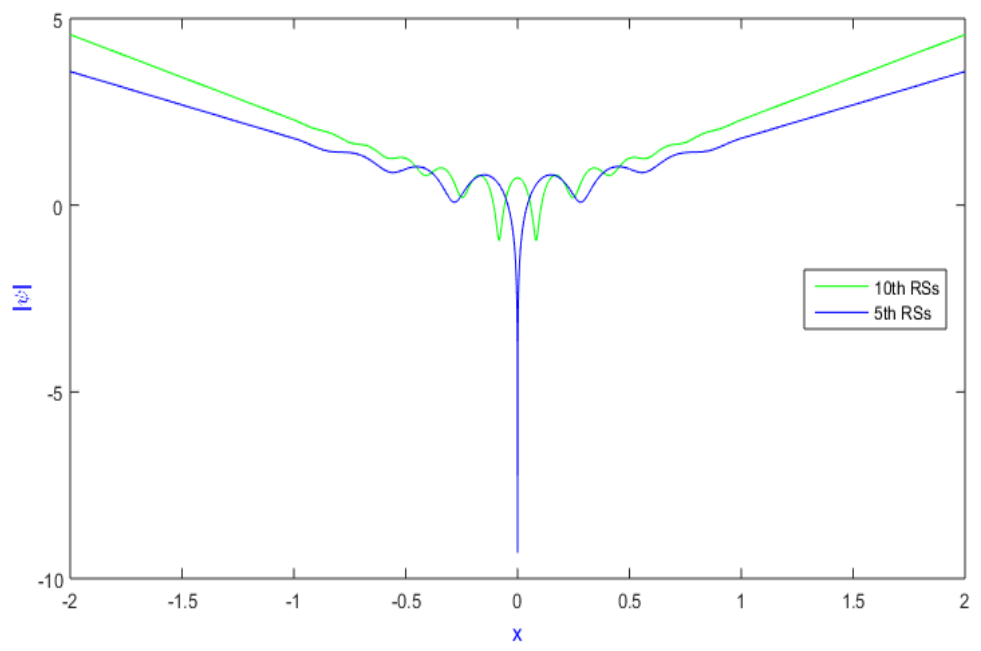

Figure 3: Plots of the absolute values of the $5^{\text {th }}$ and $10^{\text {th }} \mathrm{RSs}$ wave functions

Armitage, L. J., Doost, M. B., Langbein, W., \&Muljarov, E. A. (2014).Resonant states expansion applied to planar

Figure 2 shows the plots of the absolute values of the anti-bound state, $1^{\text {st }}, 2^{\text {nd, }}$ and $3^{\text {rd }}$ normal RSs wave functions while figure 3 shows the $5^{\text {th }}$ and $10^{\text {th }}$ normal RSs wave functions. They show a similar behavior to the bound states wave functions having a defined parity within the well. Unlike the bound states, we can see that in a normal RSs and anti-bound states there is a leak on the boundaries of the origin. We can also see that there is only one zero of $1^{\text {st }}, 3^{\text {rd, }}$ and $5^{\text {th }}$ RSs which is due to the odd nature of the wave function. However, there are two zeros of anti-bound, which are also due to the even nature of the wave function. For anti-bound states, the wave function is real while is essentially complex for normal RSs.

\section{SUMMARY AND CONCLUSION}

In this work, the concept of RSs was introduced and discussed in a one-dimensional finite square well potential. RSs were studied by seeking solutions to the time-independent Schrödinger's equation with outgoing wave boundary conditions. After the application of boundary conditions to the problem, a system of equations was generated and written in terms of secular transcendental equations for even and odd states. Solutions of these equations were analytically solved using the Newton-Raphson method in MATLAB. The full spectrum obtained includes bound states associated with pure imaginary and positive wave numbers, the antibound states associated with pure imaginary and negative wavenumbers, and the normal RSs with complex wavenumbers which lie in the lower half of the complex k-plane. The properties of RSs were considered and discussed in detail. The wave function of states of all types was plotted and compared with each other, demonstrating the probability leakage of anti-bound states and normal RSs. We demonstrate that for each RSs wave function contain asymmetric pair of states which are complex conjugate of each other.

\section{REFERENCES}

Andrew De la Bastide (2010-2011). Resonant state expansion Applied to 1-D Schrodinger Equation. Year 4 Physics report, Cardiff University, UK. waveguides.Phys.Rev. A 89, 053832.

Doost, M. B., Langbein, W., \&Muljarov, E. A. (2012).Resonant states expansion applied to planar open optical systems.Phys. Rev. A 85,023835 .

Gamow, G. (1928). Zurquantentheorie des atomkernes. Zeitschrift fur physic. 51, 204-212.

Hatano, N. (2008). Some Properties of the Resonant State in Quantum Mechanics and its Computation. Prog. Theor. Phys.119, 187.

Mandle, F. (1992). Quantum Mechanics (1 ${ }^{\text {st }}$ Edition), Wiley, 50010. Muljarov E. A., Langbein, W. \& Zimmermann, R. (2010). BrillouinWignerPerturbation theory in open electromagnetic systems. Europhys. Lett. 92, 5.

Siegert, A. J. F. (1939). On the Derivation of the Dispersion Formula forNuclear Reactions. PhysicalReview 56, 750-752.

Tanimu, A., \&Muljarov E. A. (2018). Resonant states expansion applied to one dimensional quantum systems. Physical review A. 98, 022127.

Tanimu, A., \&Muljarov, E. A. (2018). Resonant states in double and triple quantum wells.J. Phys. Commun. 2, 115008.

Uma, A.,Mashewari, \&Prema, P. (2010). Resonant states and transmission coefficient oscillations for potential wells and barriers. Am. J. Phys. 78, 412. 